\title{
TWISTED BURNSIDE-FROBENIUS THEORY FOR DISCRETE GROUPS
}

\author{
ALEXANDER FEL'SHTYN AND EVGENIJ TROITSKY
}

\begin{abstract}
It is proved for a wide class of groups including polycyclic and finitely generated polynomial growth groups that the Reidemeister number of an automorphism $\phi$ is equal to the number of finite-dimensional fixed points of the induced map $\widehat{\phi}$ on the unitary dual, if one of these numbers is finite. This theorem is a natural generalization to infinite groups of the classical Burnside-Frobenius theorem. On the other hand it has important consequences in Topological Dynamics. In some sense our theorem is a reply to a remark of J.-P. Serre.

The main technical theorems proved in the paper give a tool for a further progress.
\end{abstract}

\section{Contents}

1. Introduction

2. Preliminary considerations 4

3. Extensions and Reidemeister classes 6

4. Polycyclic and polynomial growth groups 10

5. Twisted Burnside-Frobenius theorem for RP groups 11

6. Counterexamples 12

7. Twisted conjugacy separateness 13

$\begin{array}{ll}\text { References } & 15\end{array}$

\section{INTRODUCTION}

Definition 1.1. Let $G$ be a countable discrete group and $\phi: G \rightarrow G$ an endomorphism. Two elements $x, x^{\prime} \in G$ are said to be $\phi$-conjugate or twisted conjugate, iff there exists $g \in G$ with

$$
x^{\prime}=g x \phi\left(g^{-1}\right) .
$$

We shall write $\{x\}_{\phi}$ for the $\phi$-conjugacy or twisted conjugacy class of the element $x \in G$. The number of $\phi$-conjugacy classes is called the Reidemeister number of an endomorphism $\phi$ and is denoted by $R(\phi)$. If $\phi$ is the identity map then the $\phi$-conjugacy classes are the usual conjugacy classes in the group $G$.

2000 Mathematics Subject Classification. 20C; 20E45; 22D10; 22D25; 37C25; 43A30; 46L; 47H10; $54 \mathrm{H} 25 ; 55 \mathrm{M} 20$.

Key words and phrases. Reidemeister number, twisted conjugacy classes, Burnside-Frobenius theorem, solvable group, polycyclic group, conjugacy separable group, polynomial growth, Osin group.

The second author is partially supported by RFFI Grant 05-01-00923 and Grant "Universities of Russia". 
If $G$ is a finite group, then the classical Burnside-Frobenius theorem (see e.g. [39], $[26$, p. 140]) says that the number of classes of irreducible representations is equal to the number of conjugacy classes of elements of $G$. Let $\widehat{G}$ be the unitary dual of $G$, i.e. the set of equivalence classes of unitary irreducible representations of $G$.

If $\phi: G \rightarrow G$ is an automorphism, it induces a map $\widehat{\phi}: \widehat{G} \rightarrow \widehat{G}, \widehat{\phi}(\rho)=\rho \circ \phi$. Therefore, by the Burnside-Frobenius theorem, if $\phi$ is the identity automorphism of any finite group $G$, then we have $R(\phi)=\# \operatorname{Fix}(\widehat{\phi})$.

In [6] it was discovered that this statement remains true for any automorphism $\phi$ of any finite group $G$. Indeed, consider an automorphism $\phi$ of a finite group $G$. Then $R(\phi)$ is equal to the dimension of the space of twisted invariant functions on this group. Hence, by Peter-Weyl theorem (which asserts the existence of a two-side equivariant isomorphism $\left.C^{*}(G) \cong \bigoplus_{\rho \in \widehat{G}} \operatorname{End}\left(H_{\rho}\right)\right), R(\phi)$ is identified with the sum of dimensions $d_{\rho}$ of twisted invariant elements of $\operatorname{End}\left(H_{\rho}\right)$, where $\rho$ runs over $\widehat{G}$, and the space of representation $\rho$ is denoted by $H_{\rho}$. By Schur lemma, $d_{\rho}=1$, if $\rho$ is a fixed point of $\widehat{\phi}$, and is zero otherwise. Hence, $R(\phi)$ coincides with the number of fixed points of $\widehat{\phi}$.

The attempts to generalize this theorem to the case of non-identical automorphism and of non-finite group were inspired by the dynamical questions and were the subject of a series of papers $[6,7,4,10,12,8]$.

In the present paper we introduce the following property $\mathrm{RP}$ for a countable discrete group $G$ : $\phi$-class functions of any automorphism $\phi$ with $R(\phi)<\infty$ are periodic in a natural sense (Definition 3.6).

After some preliminary and technical considerations we prove the main results of the paper, namely

(1) $\mathbf{R P}$ respects some extensions: Suppose, there is an extension $H \rightarrow G \rightarrow G / H$, where the group $H$ is a characteristic RP-group; $G / H$ is finitely generated FCgroup (i.e. a group with finite conjugacy classes). Then $G$ is an RP-group (a reformulation of Theorem 3.10).

(2) Classes of RP groups: Polycyclic groups and finitely generated groups of polynomial growth are RP-groups. Moreover, almost polycyclic groups are RP too. (Theorems 4.2, 4.4, 4.6). Twisted Burnside-Frobenius theorem is valid for them (Theorem 4.5).

(3) Twisted Burnside-Frobenius theorem for RP-groups: Let $G$ be an RP group and $\phi$ its automorphism with $R(\phi)<\infty$. Denote by $\widehat{G}_{f}$ the subset of the unitary dual $\widehat{G}$ related to finite-dimensional representations and by $S_{f}(\phi)$ the number of fixed points of $\widehat{\phi}_{f}$ on $\widehat{G}_{f}$. Then $R(\phi)=S_{f}(\phi)$. (Theorem 5.2).

(4) Twisted Burnside-Frobenius theorem for almost polycyclic groups: Let $G$ be an almost polycyclic group. Then $R(\phi)=S_{f}(\phi)$ if one of these numbers is finite (Theorem 7.8).

In some sense our theory is a reply to a remark of J.-P. Serre [39, (d), p.34] that for compact infinite groups an analogue of Burnside-Frobenius theorem is not interesting: $\infty=\infty$. It turns out that for infinite discrete groups the situation differs significantly, and even in non-twisted situations the number of classes can be finite (for one of the first examples see another book of J.-P. Serre [40]). A number of examples of groups and 
automorphisms with finite Reidemeister numbers was obtained and studied in $[4,16,14$, $12,8]$.

Using the same argument as in [10] one obtains from the twisted Burnside-Frobenius theorem the following dynamical and number-theoretical consequence which, together with the twisted Burnside-Frobenius theorem itself, is very important for the realization problem of Reidemeister numbers in topological dynamics and the study of the Reidemeister zeta-function.

Let $\mu(d), d \in \mathbb{N}$, be the Möbius function, i.e.

$$
\mu(d)= \begin{cases}1 & \text { if } d=1, \\ (-1)^{k} & \text { if } d \text { is a product of } k \text { distinct primes } \\ 0 & \text { if } d \text { is not square }- \text { free. }\end{cases}
$$

Congruences for Reidemeister numbers: Let $\phi: G \rightarrow G$ be an automorphism of a countable discrete RP-group $G$ such that all numbers $R\left(\phi^{n}\right)$ are finite. Then one has for all $n$,

$$
\sum_{d \mid n} \mu(d) \cdot R\left(\phi^{n / d}\right) \equiv 0 \bmod n
$$

These theorems were proved previously in a number of special cases in $[6,7,10,12,8]$. We would like to emphasize the following important remarks.

(1) In the original formulation by Fel'shtyn and Hill [6] the conjecture about twisted Burnside-Frobenius theorem asserts an equality of $R(\phi)$ and the number of fixed points of $\widehat{\phi}$ on $\widehat{G}$. This conjecture was proved in $[6,10]$ for f.g. type I groups.

(2) As it follows from a key example, which we have studied with A. Vershik in [12], an RP-group can have infinite dimensional "supplementary" fixed representations. More precisely we discuss in that paper the case of a semi-direct product of the action of $\mathbb{Z}$ on $\mathbb{Z} \oplus \mathbb{Z}$ by a hyperbolic automorphism with finite Reidemeister number (four to be precise) and the number of fixed points of $\widehat{\phi}$ on $\widehat{G}$ equal or greater than five, while the number of fixed points on $\widehat{G}_{f}$ is four.

This gives a counterexample to the conjecture in its original formulation and leads to the formulation using only fixed points in $\widehat{G}_{f}$. The new conjecture is proved in the present paper for a wide class of f.g. groups.

(3) The origin of the phenomenon of an extra fixed point lies in bad separation properties of $\widehat{G}$ for general discrete groups. A more deep study leads to the following general theorem.

WEAK TWISTED BuRnside THEOREM [43]: The number $R_{*}(\phi)$ of Reidemeister classes related to twisted invariant functions on $G$ from the Fourier-Stieltjes algebra $B(G)$ is equal to the number $S_{*}(\phi)$ of generalized fixed points of $\widehat{\phi}$ on the Glimm spectrum of $G, i$. e. on the complete regularization of $\widehat{G}$, if one of $R_{*}(\phi)$ and $S_{*}(\phi)$ is finite.

The argument is based on a non-commutative version of the well-known Riesz(Markov-Kakutani) theorem, which identifies the space of linear functionals on algebra $A=C(X)$ and the space of regular measures on $X$. To prove the weak twisted Burnside theorem we first obtain a generalization of this theorem to the case of non-commutative $C^{*}$-algebra $A$ via Dauns-Hofmann sectional representation theorem (in the same paper [43]). The corresponding measures on Glimm 
spectrum are functional-valued. In extreme situation this theorem is tautological, but for group $C^{*}$-algebras of discrete groups in many cases one obtains some new tool for counting twisted conjugacy classes. This is a basis for an approach to the problem which is an alternative one to the approach developed in the present paper.

(4) In fact the main Theorem 3.10 allows to verify periodicity of $\phi$-class functions in a number of cases which are not in the classes described in Section 4. Nevertheless for pathological groups from Section 6 even the modified conjecture is not true. Keeping in mind that for Gromov hyperbolic groups $R(\phi)$ is always infinite (as well as for Baumslag-Solitar groups and some generalizations, cf. [13, 29, 5, 28, 42]) while in the "opposite" case the twisted Burnside theorem is proved, we can hope that various use of Theorem 3.10 can lead to a complete resolution of the problem, if the groups from Section 6 will be handled.

The interest in twisted conjugacy relations has its origins, in particular, in the NielsenReidemeister fixed point theory (see, e.g. [24, 4]), in Selberg theory (see, eg. [41, 1]), and Algebraic Geometry (see, e.g. [20]).

Concerning some topological applications of our main results, they are already described in [10]. The congruences give some necessary conditions for the realization problem for Reidemeister numbers in topological dynamics. The relations with Selberg theory will be presented in a forthcoming paper.

Acknowledgement. The present research is a part of our joint research programm in Max-Planck-Institut für Mathematik (MPI) in Bonn. We would like to thank the MPI for its kind support and hospitality while the most part of this work has been completed.

The authors are grateful to R. Hill, M. Sapir, A. Shtern, L. Vainerman, A. Vershik for helpful discussions.

The results of Sections 2, 4, 6, and 7 are obtained by the authors jointly, the results of Sections 3 and 5 are obtained by E. Troitsky.

\section{Preliminary CONSIDERAtions}

The following fact will be useful.

Theorem 2.1 ([36, Theorem 1.41]). If $G$ is a finitely generated group and $H$ is a subgroup with finite index in $G$, then $H$ is finitely generated.

Lemma 2.2. Let $G$ be finitely generated, and $H^{\prime} \subset G$ its subgroup of finite index. Then there is a characteristic subgroup $H \subset G$ of finite index, $H \subset H^{\prime}$.

Proof. Since $G$ is finitely generated, there is only finitely many subgroups of the same index as $H^{\prime}$ (see [22], [27, $\left.\S 38\right]$ ). Let $H$ be their intersection. Then $H$ is characteristic, in particular normal, and of finite index.

Lemma 2.3. Let $G$ be abelian. The twisted conjugacy class $H$ of $e$ is a subgroup. The other ones are cosets $g H$.

Proof. The first statement follows from the equalities

$$
h \phi\left(h^{-1}\right) g \phi\left(g^{-1}\right)=g h \phi\left((g h)^{-1}, \quad\left(h \phi\left(h^{-1}\right)\right)^{-1}=\phi(h) h^{-1}=h^{-1} \phi(h) .\right.
$$


For the second statement suppose $a \sim b$, i.e. $b=h a \phi\left(h^{-1}\right)$. Then

$$
g b=g h a \phi\left(h^{-1}\right)=h(g a) \phi\left(h^{-1}\right), \quad g b \sim g a .
$$

Let us denote by $\tau_{g}: G \rightarrow G$ the automorphism $\tau_{g}(\widetilde{g})=g \widetilde{g} g^{-1}$ for $g \in G$. Its restriction on a normal subgroup we will denote by $\tau_{g}$ as well.

Lemma 2.4. $\{g\}_{\phi} k=\{g k\}_{\tau_{k-1} \circ \phi}$.

Proof. Let $g^{\prime}=f g \phi\left(f^{-1}\right)$ be $\phi$-conjugate to $g$. Then

$$
g^{\prime} k=f g \phi\left(f^{-1}\right) k=f g k k^{-1} \phi\left(f^{-1}\right) k=f(g k)\left(\tau_{k^{-1}} \circ \phi\right)\left(f^{-1}\right) .
$$

Conversely, if $g^{\prime}$ is $\tau_{k^{-1}} \circ \phi$-conjugate to $g$, then

$$
g^{\prime} k^{-1}=f g\left(\tau_{k^{-1}} \circ \phi\right)\left(f^{-1}\right) k^{-1}=f g k^{-1} \phi\left(f^{-1}\right) .
$$

Hence a shift maps $\phi$-conjugacy classes onto classes related to another automorphism.

Corollary 2.5. $R(\phi)=R\left(\tau_{g} \circ \phi\right)$.

Theorem 2.6 (see [24]). Let $A$ be a finitely generated Abelian group, $\psi: A \rightarrow A$ its automorphism. Then $R(\psi)=\# \operatorname{Coker}(\psi-\mathrm{Id})$, i.e. to the index of subgroup generated by elements of the form $x^{-1} \psi(x)$.

Proof. By Lemma 2.3, $R(\psi)$ is equal to the index of the subgroup $H=\{e\}_{\psi}$. This group consists by definition of elements of the form $x^{-1} \psi(x)$.

The following construction relates $\phi$-conjugacy classes and some conjugacy classes of another group. It was obtained in topological context by Boju Jiang and Laixiang Sun in [25]. Consider the action of $\mathbb{Z}$ on $G$, i.e. a homomorphism $\mathbb{Z} \rightarrow \operatorname{Aut}(G), n \mapsto \phi^{n}$. Let $\Gamma$ be a corresponding semi-direct product $\Gamma=G \rtimes \mathbb{Z}$ :

$$
\Gamma:=<G, t \mid \operatorname{tgt}^{-1}=\phi(g)>
$$

in terms of generators and relations, where $t$ is a generator of $\mathbb{Z}$. The group $G$ is a normal subgroup of $\Gamma$. As a set, $\Gamma$ has the form

$$
\Gamma=\sqcup_{n \in \mathbb{Z}} G \cdot t^{n},
$$

where $G \cdot t^{n}$ is the coset by $G$ containing $t^{n}$.

Remark 2.7. Any usual conjugacy class of $\Gamma$ is contained in some $G \cdot t^{n}$. Indeed, $g g^{\prime} t^{n} g^{-1}=g g^{\prime} \phi^{n}\left(g^{-1}\right) t^{n}$ and $t g^{\prime} t^{n} t^{-1}=\phi\left(g^{\prime}\right) t^{n}$.

Lemma 2.8. Two elements $x, y$ of $G$ are $\phi$-conjugate iff $x t$ and $y t$ are conjugate in the usual sense in $\Gamma$. Therefore $g \mapsto g \cdot t$ is a bijection from the set of $\phi$-conjugacy classes of $G$ onto the set of conjugacy classes of $\Gamma$ contained in $G \cdot t$.

Proof. If $x$ and $y$ are $\phi$-conjugate then there is a $g \in G$ such that $g x=y \phi(g)$. This implies $g x=y t g t^{-1}$ and therefore $g(x t)=(y t) g$ so $x t$ and $y t$ are conjugate in the usual sense in $\Gamma$. Conversely, suppose $x t$ and $y t$ are conjugate in $\Gamma$. Then there is a $g t^{n} \in \Gamma$ with $g t^{n} x t=y t g t^{n}$. From the relation $t x t^{-1}=\phi(x)$ we obtain $g \phi^{n}(x) t^{n+1}=y \phi(g) t^{n+1}$ and therefore $g \phi^{n}(x)=y \phi(g)$. Hence, $y$ and $\phi^{n}(x)$ are $\phi$-conjugate. Thus, $y$ and $x$ are $\phi$-conjugate, because $x$ and $\phi(x)$ are always $\phi$-conjugate: $\phi(x)=x^{-1} x \phi(x)$. 


\section{Extensions and Reidemeister Classes}

Consider a group extension respecting homomorphism $\phi$ :

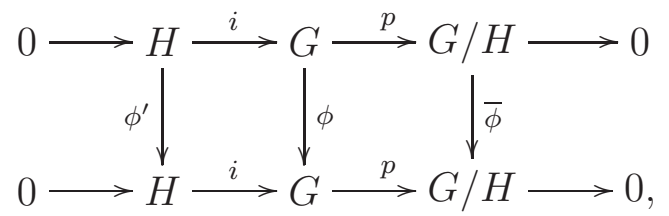

where $H$ is a normal subgroup of $G$. The argument below, especially related the role of fixed points, has a partial intersection with $[16,17]$.

First of all let us notice that the Reidemeister classes of $\phi$ in $G$ are mapped epimorphically on classes of $\bar{\phi}$ in $G / H$. Indeed,

$$
p(\widetilde{g}) p(g) \bar{\phi}\left(p\left(\widetilde{g}^{-1}\right)\right)=p\left(\widetilde{g} g \phi\left(\widetilde{g}^{-1}\right) .\right.
$$

Suppose, $R(\phi)<\infty$. Then the previous remark implies $R(\bar{\phi})<\infty$. Consider a class $D=\{h\}_{\tau_{g} \phi^{\prime}}$, where $\tau_{g}(h):=g h g^{-1}, g \in G, h \in H$. The corresponding equivalence relation is

$$
h \sim \widetilde{h} h g \phi^{\prime}\left(\widetilde{h}^{-1}\right) g^{-1} .
$$

Since $H$ is normal, the automorphism $\tau_{g}: H \rightarrow H$ is well defined. We will denote by $D$ the image $i D$ as well. By (5) the shift $D g$ is a subset of $H g$ is characterized by

$$
h g \sim \widetilde{h}(h g) \phi^{\prime}\left(\widetilde{h}^{-1}\right) .
$$

Hence it is a subset of $\{h g\}_{\phi} \cap H g$ and the partition $H g=\cup\left(\{h\}_{\tau_{g} \phi^{\prime}}\right) g$ is a subpartition of $H g=\cup\left(H g \cap\{h g\}_{\phi}\right)$.

We need the following statements.

Lemma 3.1. Suppose, the extension (3) satisfies the following conditions:

(1) $\# \operatorname{Fix} \bar{\phi}=k<\infty$,

(2) $R(\phi)<\infty$.

Then

$$
R\left(\phi^{\prime}\right) \leq k \cdot(R(\phi)-R(\bar{\phi})+1) .
$$

If $G / H$ is abelian, let $g_{i}$ be some elements with $p\left(g_{i}\right)$ being representatives of all different $\bar{\phi}$-conjugacy classes, $i=1, \ldots, R(\bar{\phi})$. Then

$$
\sum_{i=1}^{R(\bar{\phi})} R\left(\tau_{g_{i}} \phi^{\prime}\right) \leq k \cdot R(\phi) .
$$

Proof. Consider classes $\{z\}_{\phi^{\prime}}, z \in G$, i.e. the classes of relation $z \sim h z \phi^{\prime}\left(h^{-1}\right), h \in H$. The group $G$ acts on them by $z \mapsto g z \phi\left(g^{-1}\right)$. Indeed,

$$
\begin{aligned}
g\left[\widetilde{h} h \phi\left(\widetilde{h}^{-1}\right)\right] \phi\left(g^{-1}\right)=\left(g \widetilde{h} g^{-1}\right)\left(g h \phi\left(g^{-1}\right)\right) & \left(\phi(g) \phi\left(\widetilde{h}^{-1}\right) \phi\left(g^{-1}\right)\right) \\
& =\left(g \widetilde{h} g^{-1}\right)\left(g h \phi\left(g^{-1}\right)\right) \phi\left(g \widetilde{h} g^{-1}\right) \in\left\{g h \phi\left(g^{-1}\right)\right\}_{\phi^{\prime}},
\end{aligned}
$$

because $H$ is normal and $g \widetilde{h} g^{-1} \in H$. Due to invertibility, this action of $G$ transposes classes $\{z\}_{\phi^{\prime}}$ inside one class $\{g\}_{\phi}$. Hence, the number $d$ of classes $\{h\}_{\phi^{\prime}}$ inside $\{h\}_{\phi} \cap H$ 
does not exceed the number of $g \in G$ such that $p(g) \bar{\phi}\left(p\left(g^{-1}\right)\right)=\bar{e}$. Since two elements $g$ and $g h$ in one $H$-coset induce the same permutation of classes $\{h\}_{\phi^{\prime}}$, the mentioned number $d$ does not exceed the number of $z \in G / H$ such that $z \bar{\phi}\left(z^{-1}\right)=\bar{e}$, i.e. $d \leq k$. This implies (7).

Now we discuss $\phi$-classes over $\bar{\phi}$-classes other than $\{\bar{e}\}_{\bar{\phi}}$ for an abelian $G / H$. An estimation analogous to the above one leads to the number of $z \in G / H$ such that $z z_{0} \bar{\phi}\left(z^{-1}\right)=z_{0}$ for some fixed $z_{0}$. But for an Abelian $G / H$ they form the same group $\operatorname{Fix}(\bar{\phi})$. This together with the description (6) of shifts of $D$ at the beginning of the present Section implies (8).

Lemma 3.2. Suppose, in the extension (3) the group $H$ is abelian. Then \# $\operatorname{Fix}(\phi) \leq$ $\# \operatorname{Fix}\left(\phi^{\prime}\right) \cdot \# \operatorname{Fix}(\bar{\phi})$.

Proof. Let $s: G / H \rightarrow G$ be a section of $p$. If $s(z) h$ is a fixed point of $\phi$ then

$$
(s(z))^{-1} \phi(s(z))=h \phi^{\prime}\left(h^{-1}\right) .
$$

Hence, $z \in \operatorname{Fix}(\bar{\phi})$ and left hand side takes $k:=\# \operatorname{Fix}(\bar{\phi})$ values $h_{1}, \ldots, h_{k}$. Let us estimate the number of $s(z) h$ for a fixed $z$ such that $(s(z))^{-1} \phi(s(z))=h_{i}$. These $h$ have to satisfy (9). Since $H$ is abelian, if one has

$$
h_{i}=h \phi^{\prime}\left(h^{-1}\right)=\widetilde{h} \phi^{\prime}\left(\widetilde{h}^{-1}\right),
$$

then $h^{-1} \widetilde{h} \in \operatorname{Fix}\left(\phi^{\prime}\right)$ and we are done.

Theorem 3.3. Let $A$ be a finitely generated Abelian group, $\psi: A \rightarrow A$ its automorphism with $R(\psi)<\infty$. Then \# $\operatorname{Fix}(\psi)<\infty$.

Moreover, $R(\psi) \geq \#$ Fix $(\psi)$.

Proof. Let $T$ be the torsion subgroup. It is finite and characteristic. We obtain the extension $T \rightarrow A \rightarrow A / T$ respecting $\phi$. Since $A / T \cong \mathbb{Z}^{k}, \operatorname{Fix}(\bar{\psi}: A / T \rightarrow A / T)=\bar{e}$, by [24],[4, Sect. 2.3.1]. Hence, by Lemma 3.2, \# $\operatorname{Fix}(\psi) \leq \# \operatorname{Fix}\left(\psi^{\prime}\right), \psi^{\prime}: T \rightarrow T$. For any finite abelian group $T$ one clearly has $\# \operatorname{Fix}\left(\psi^{\prime}\right)=R\left(\psi^{\prime}\right)$ by Theorem 2.6 (cf. [4, p. 7]). Finally, $R\left(\psi^{\prime}\right) \leq R(\psi)$ by $(8)$.

Let us remind the following definitions of a class of groups.

Definition 3.4. A group with finite conjugacy classes is called FC-group.

In a FC-group the elements of finite order form a characteristic subgroup with locally infinite abelian factor group; a finitely generated FC-group contains in its center a free abelian group of finite index in the whole group [31].

Lemma 3.5. An automorphism $\phi$ of a finitely generated FC-group $G$ with $R(\phi)<\infty$ has a finite number of fixed points.

The same is true for $\tau_{x} \circ \phi$. Hence, the number of $g \in G$ such that for some $x \in G$

$$
g x \phi\left(g^{-1}\right)=x
$$

is still finite. 
Proof. Let $A$ be the center of $G$. As it was indicated, $A$ has a finite index in $G$ and hence, by Theorem 2.1, is f.g. Since $A$ is characteristic, one has an extension $A \rightarrow G \rightarrow G / A$ respecting $\phi$. One has $R\left(\phi^{\prime}\right) \leq R(\phi) \cdot|G / A|$ by Lemma 3.1 and \# $\operatorname{Fix}\left(\phi^{\prime}\right) \leq R(\phi) \cdot|G / A|$ by Theorem 3.3. Then \# $\operatorname{Fix}(\phi) \leq R(\phi) \cdot|G / A|^{2}$ by Lemma 3.2.

Definition 3.6. We say that a group $G$ has the property RP if for any automorphism $\phi$ with $R(\phi)<\infty$ the characteristic functions $f$ of REIDEMEISTER classes (hence all $\phi$-central functions) are PERIODIC in the following sense.

There exists a finite group $K$, its automorphism $\phi_{K}$, and epimorphism $F: G \rightarrow K$ such that

(1) The diagram



commutes.

(2) $f=F^{*} f_{K}$, where $f_{K}$ is a characteristic function of a subset of $K$.

If this property holds for a concrete automorphism $\phi$, we will denote this by $\operatorname{RP}(\phi)$.

Remark 3.7. By (2) there is only one class $\{g\}_{\phi}$ which maps onto $\{F(g)\}_{\phi_{K}}$. Hence, $F$ induces a bijection of Reidemeister classes.

Lemma 3.8. Suppose, $G$ is f.g. and $R(\phi)<\infty$. Then characteristic functions of $\phi$ conjugacy classes are periodic (i.e. $G$ has $\mathrm{RP}(\phi))$ if and only if their left shifts generate a finite dimensional space.

Proof. From the supposition of finite dimension it follows that the stabilizer of each $\phi$ conjugacy class has finite index. Hence, the common stabilizer of all $\phi$-conjugacy classes under left shifts is an intersection of finitely many subgroups, each of finite index. Hence, its index is finite. By Lemma 2.2 there is some smaller subgroup $G_{S}$ of finite index which is normal and $\phi$-invariant. Then one can take $K=G / G_{S}$. Indeed, it is sufficient to verify that the projection $F$ is one to one on classes. In other words, that each coset of $G_{s}$ enters only one $\phi$-conjugacy class, or any two elements of coset are $\phi$-conjugated. Consider $g$ and $h g, g \in G, h \in G_{s}$. Since $h$ by definition preserves classes, $h g=x g \phi\left(x^{-1}\right)$ for some $x \in G$, as desired.

Conversely, if $G$ has $\operatorname{RP}(\phi)$, the class $\{g\}_{\phi}$ is a full pre-image $F^{-1}(S)$ of some class $S \subset K$. Then its left shift can be described as

$$
\begin{aligned}
g^{\prime}\{g\}_{\phi} & =g^{\prime} F^{-1}(S)=\left\{g^{\prime} g_{1} \mid g_{1} \in F^{-1}(S)\right\}=\left\{g \mid\left(g^{\prime}\right)^{-1} g \in F^{-1}(S)\right\} \\
& =\left\{g \mid F\left(\left(g^{\prime}\right)^{-1} g\right) \in S\right\}=\left\{g \mid F(g) \in F\left(g^{\prime}\right)(S)\right\}=F^{-1}\left(F\left(g^{\prime}\right)(S)\right) .
\end{aligned}
$$

Since $K$ is finite, the number of these sets is finite.

Remark 3.9. 1) In this situation in accordance with Lemma 2.2 the subgroup $G_{S}$ is characteristic, i.e. invariant under any automorphism.

2) Also, the group $G / G_{S}$ will serve as $K$ (i.e. give rise a bijection on sets of Reidemeister classes) not only for $\phi$ but for $\tau_{g} \circ \phi$ for any $g \in G$ because they have the same collection of left shifts of Reidemeister classes by Lemma 2.4 . 
Theorem 3.10. Suppose, the extension (3) satisfies the following conditions:

(1) $H$ has RP;

(2) $G / H$ is FC f.g.

Then $G$ has $\operatorname{RP}(\phi)$.

Proof. We have $R(\bar{\phi})<\infty$, hence \# Fix $(\bar{\phi})<\infty$ by Lemma 3.5 as well as $\# \operatorname{Fix}\left(\tau_{z} \bar{\phi}\right)<\infty$ for any $z \in G / H$. Then by Lemma $3.1 R\left(\tau_{g} \phi^{\prime}\right)<\infty$ for any $g \in G$. Let $g_{1}, \ldots, g_{s}$, $s=R(\bar{\phi})$, be elements of $G$ which are mapped by $p$ to different Reidemeister classes of $\bar{\phi}$. Now we can apply the supposition that $H$ has RP and find a characteristic subgroup $H_{K}:=\operatorname{Ker} F \subset H$ of finite index such that $F: H \rightarrow K$ gives rise a bijection for Reidemeister classes of each $\tau_{g_{i}} \circ \phi^{\prime}, i=1, \ldots, s$, moreover, it is contained in the stabilizer of each twisted conjugacy class of each of these automorphisms. In particular, it is normal in $G$. Hence, we can take a quotient by $H_{K}$ of the extension (3):

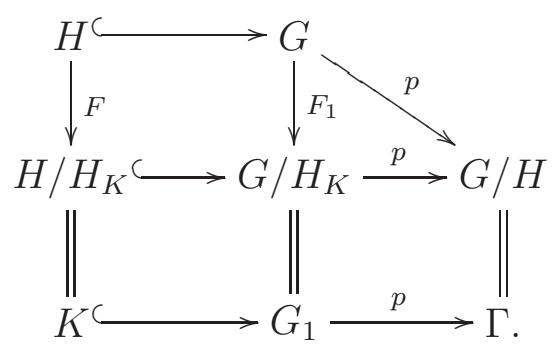

The quotient map $F_{1}: G \rightarrow G / H_{K}$ takes $\{g\}_{\phi}$ to $\{g\}_{\phi}$ and it is a unique class with this property (we conserve the notations $e, g, \phi$ for the quotient objects). Indeed, suppose two classes are mapped onto one. This means that there are two elements $g$ and $g h_{K}$ of these different classes, $h_{K} \in H_{K}$. One can choose $\widetilde{g} \in G$ such that $\widetilde{g} g \phi\left(\widetilde{g}^{-1}\right)=g_{i} h$ for some $i=1, \ldots, s, h \in H$. Then

$$
\widetilde{g}\left(g h_{K}\right) \phi\left(\widetilde{g}^{-1}\right)=\widetilde{g} \widetilde{g}^{-1} g_{i} h \phi(\widetilde{g}) h_{K} \phi\left(\widetilde{g}^{-1}\right)=g_{i} h \widehat{h}_{K}, \quad \widehat{h}_{K} \in H_{K} .
$$

Hence, $g_{i} h$ and $g_{i} h \widehat{h}_{K}$ belong to the same (different) classes as $g$ and $g h_{K}$. Moreover, they can not be $\phi$-conjugate by elements of $H$. Hence (cf. (6)), the elements $h$ and $h \widehat{h}_{K}$ are not $\left(\tau_{g_{i}} \circ \phi^{\prime}\right)$-conjugate in $H$. But this contradicts the choice of $H_{K} \ni \widehat{h}_{K}$.

By Lemma 3.8 (applied to $G_{1}$ and concrete automorphism $\phi$ ) for the purpose to find a map $F_{2}: G_{1} \rightarrow K_{1}$ with properties (1) and (2) of the Definition 3.6 it is sufficient to verify that shifts of the characteristic function of $\{h\}_{\phi} \subset G_{1}$ form a finite dimensional space, i.e. the shifts of $\{h\}_{\phi} \subset G_{1}$ form a finite collection of subsets of $G_{1}$. After that one can take the composition

$$
G \stackrel{F_{1}}{\longrightarrow} G_{1} \stackrel{F_{2}}{\longrightarrow} K_{1}
$$

to complete the proof of theorem.

Let us observe, that we can apply Lemma 3.8 because the group $G_{1}$ is finitely generated: we can take as generators all elements of $K$ and some pre-images $s\left(z_{i}\right) \in G_{1}$ under $p$ of a finite system of generators $z_{i}$ for $\Gamma$. Indeed, for any $x \in G_{1}$ one can find some product of $z_{i}$ to be equal to $p(x)$. Then the same product of $s\left(z_{i}\right)$ differs from $x$ by an element of $K$.

Let us prove that the mentioned space of shifts is finite-dimensional. By Lemma 2.4 these shifts of $\{h\}_{\phi} \subset G_{1}$ form a subcollection of

$$
\{x\}_{\tau_{y} \circ \phi}, \quad x, y \in G_{1} .
$$


Hence, by Corollary 2.5 it is sufficient to verify that the number of different automorphisms $\tau_{y}: G_{1} \rightarrow G_{1}$ is finite.

Let $x_{1}, \ldots, x_{n}$ be some generators of $G_{1}$. Then the number of different $\tau_{y}$ does not exceed

$$
\prod_{j=1}^{n} \#\left\{\tau_{y}\left(x_{j}\right) \mid y \in G_{1}\right\} \leq \prod_{j=1}^{n}|K| \cdot \#\left\{\tau_{z}\left(p\left(x_{j}\right)\right) \mid z \in \Gamma\right\},
$$

where the last numbers are finite by the definition of $\mathrm{FC}$ for $\Gamma$.

\section{Polycyclic and polynomial growth Groups}

Now we describe using Theorem 3.10 some classes of RP groups. Of course these classes are only a small part of possible corollaries of this theorem.

Let $G^{\prime}=[G, G]$ be the commutator subgroup or derived group of $G$, i.e. the subgroup generated by commutators. $G^{\prime}$ is invariant under any homomorphism, in particular it is normal. It is the smallest normal subgroup of $G$ with an abelian factor group. Denoting $G^{(0)}:=G, G^{(1)}:=G^{\prime}, G^{(n)}:=\left(G^{(n-1)}\right)^{\prime}, n \geq 2$, one obtains derived series of $G$ :

$$
G=G^{(0)} \supset G^{\prime} \supset G^{(2)} \supset \cdots \supset G^{(n)} \supset \ldots
$$

If $G^{(n)}=e$ for some $n$, i.e. the series (10) stabilizes by trivial group, the group $G$ is solvable;

Definition 4.1. A solvable group with derived series with cyclic factors is called polycyclic group.

Theorem 4.2. Any polycyclic group is RP.

Proof. By Lemma 2.3 any commutative group is RP. Any extension with $H$ being the commutator subgroup $G^{\prime}$ of $G$ respects any automorphism $\phi$ of $G$, because $G^{\prime}$ is evidently characteristic. The factor group is abelian, in particular FC.

Since any polycyclic group is a result of finitely many such extensions with finitely generated (cyclic) factor groups, starting from Abelian group, applying inductively Theorem 3.10 we obtain the result.

Theorem 4.3. Any finitely generated nilpotent group is RP.

Proof. These groups are supersolvable, hence, polycyclic [37, 5.4.6, 5.4.12].

Theorem 4.4. Any finitely generated group of polynomial growth is RP.

Proof. By [19] a finitely generated group of polynomial growth is just as finite extension of a f.g. nilpotent group $H$. The subgroup $H$ can be supposed to be characteristic, i.e. $\phi(H)=H$ for any automorphism $\phi: G \rightarrow G$. Indeed, let $H^{\prime} \subset G$ be a nilpotent subgroup of index $j$. Let $H$ be the subgroup from Lemma 2.2. By Theorem 2.1 it is finitely generated. Also, it is nilpotent as a subgroup of nilpotent group (see $[27, \S 26]$ ).

Since a finite group is a particular case of FC group and f.g. nilpotent group has RP by Theorem 4.3, we can apply Theorem 3.10 to complete the proof.

The proof of the following twisted Burnside-Frobenius theorem can be extracted from Theorem 5.2, but we formulate it separately due to importance of the classes under consideration. 
Theorem 4.5. The Reidemeister number of any automorphism $\phi$ of a f.g. group of polynomial growth or polycyclic group with $R(\phi)<\infty$ is equal to the number of finitedimensional fixed points of $\widehat{\phi}$ on the unitary dual of this group.

Theorem 4.6. Any almost polycyclic group, i.e. an extension of a polycyclic group with a finite factor group, is RP.

Proof. The proof repeats almost literally the proof of Theorem 4.4. One has to use the fact that a subgroup of a polycyclic group is polycyclic [37, p. 147].

These theorems will be proved below in a more strong form (Theorem 7.8).

\section{Twisted Burnside-Frobenius theOrem For RP Groups}

Definition 5.1. Denote by $\widehat{G}_{f}$ the subset of the unitary dual $\widehat{G}$ related to finite-dimensional representations.

Theorem 5.2 (Twisted Burnside-Frobenius Theorem for RP-groups). Let $G$ be an RP group and $\phi$ its automorphism with $R(\phi)<\infty$. Denote by $S_{f}(\phi)$ the number of fixed points of $\widehat{\phi}_{f}$ on $\widehat{G}_{f}$. Then

$$
R(\phi)=S_{f}(\phi)
$$

Proof. The coefficients of finite-dimensional non-equivalent irreducible representations of $G$ are linear independent by Frobenius-Schur theorem (see $[2,(27.13)]$ ). Moreover, the coefficients of non-equivalent unitary finite-dimensional irreducible representations are orthogonal to each other as functions on the universal compact group associated with the initial group $[3,16.1 .3]$ by the Peter-Weyl theorem. Hence, their linear combinations are orthogonal to each other as well.

It is sufficient to verify the following three statements:

1) If $R(\phi)<\infty$, than each $\phi$-class function is a finite linear combination of twistedinvariant functionals being coefficients of points of Fix $\widehat{\phi}_{f}$.

2) If $\rho \in \operatorname{Fix} \widehat{\phi}_{f}$, there exists one and only one (up to scaling) twisted invariant functional on $\rho\left(C^{*}(G)\right)$ (this is a finite full matrix algebra).

3) For different $\rho$ the corresponding $\phi$-class functions are linearly independent. This follows from the remark at the beginning of the proof.

Let us remark that the property RP implies in particular that $\phi$-central functions (for $\phi$ with $R(\phi)<\infty)$ are functionals on $C^{*}(G)$, not only $L^{1}(G)$, i.e. are in the Fourier-Stieltijes algebra $B(G)$.

The statement 1) follows from the RP property. Indeed, this $\phi$-class function $f$ is a linear combination of functionals coming from some finite collection $\left\{\rho_{i}\right\}$ of elements of $\widehat{G}_{f}$ (these representations $\rho_{1}, \ldots, \rho_{s}$ are in fact representations of the form $\pi_{i} \circ F$, where $\pi_{i}$ are irreducible representations of the finite group $K$ and $F: G \rightarrow K$, as in the definition of RP). So,

$$
f=\sum_{i=1}^{s} f_{i} \circ \rho_{i}, \quad \rho_{i}: G \rightarrow \operatorname{End}\left(V_{i}\right), \quad f_{i}: \operatorname{End}\left(V_{i}\right) \rightarrow \mathbb{C}, \quad \rho_{i} \neq \rho_{j},(i \neq j) .
$$


For any $g, \widetilde{g} \in G$ one has

$$
\sum_{i=1}^{s} f_{i}\left(\rho_{i}(\widetilde{g})\right)=f(\widetilde{g})=f\left(g \widetilde{g} \phi\left(g^{-1}\right)\right)=\sum_{i=1}^{s} f_{i}\left(\rho_{i}\left(g \widetilde{g} \phi\left(g^{-1}\right)\right)\right) .
$$

By the observation at the beginning of the proof concerning linear independence,

$$
f_{i}\left(\rho_{i}(\widetilde{g})\right)=f_{i}\left(\rho_{i}\left(g \widetilde{g} \phi\left(g^{-1}\right)\right)\right) . \quad i=1, \ldots, s,
$$

i.e. $f_{i}$ are twisted-invariant. For any $\rho \in \widehat{G}_{f}, \rho: G \rightarrow \operatorname{End}(V)$, any functional $\omega$ : $\operatorname{End}(V) \rightarrow \mathbb{C}$ has the form $a \mapsto \operatorname{Tr}(b a)$ for some fixed $b \in \operatorname{End}(V)$. Twisted invariance implies twisted invariance of $b$ (evident details can be found in [10, Sect. 3]). Hence, $b$ is intertwining between $\rho$ and $\rho \circ \phi$ and $\rho \in \operatorname{Fix}\left(\widehat{\phi}_{f}\right)$. The uniqueness of intertwining operator (up to scaling) implies 2).

\section{Counterexamples}

Now let us present some counterexamples to this statement for pathological (monster) discrete groups. Suppose, an infinite discrete group $G$ has a finite number of conjugacy classes. Such examples can be found in [40] (HNN-group), [32, p. 471] (Ivanov group), and [33] (Osin group). Then evidently, the characteristic function of unity element is not almost-periodic and the argument above is not valid. Moreover, let us show, that these groups give rise counterexamples to above theorem.

Example 6.1. For the Osin group the Reidemeister number $R(\mathrm{Id})=2$, while there is only trivial (1-dimensional) finite-dimensional representation. Indeed, Osin group is an infinite finitely generated group $G$ with exactly two conjugacy classes. All nontrivial elements of this group $G$ are conjugate. So, the group $G$ is simple, i.e. $G$ has no nontrivial normal subgroup. This implies that group $G$ is not residually finite (by definition of residually finite group). Hence, it is not linear (by Mal'cev theorem [30], [37, 15.1.6]) and has no finite-dimensional irreducible unitary representations with trivial kernel. Hence, by simplicity of $G$, it has no finite-dimensional irreducible unitary representation with nontrivial kernel, except of the trivial one.

Let us remark that Osin group is non-amenable, contains the free group in two generators $F_{2}$, and has exponential growth.

Example 6.2. For large enough prime numbers $p$, the first examples of finitely generated infinite periodic groups with exactly $p$ conjugacy classes were constructed by Ivanov as limits of hyperbolic groups (although hyperbolicity was not used explicitly) (see [32, Theorem 41.2]). Ivanov group $G$ is infinite periodic 2-generator group, in contrast to the Osin group, which is torsion free. The Ivanov group $G$ is also a simple group. The proof (kindly explained to us by M. Sapir) is the following. Denote by $a$ and $b$ the generators of $G$ described in [32, Theorem 41.2]. In the proof of Theorem 41.2 on [32] it was shown that each of elements of $G$ is conjugate in $G$ to a power of generator $a$ of order $s$. Let us consider any normal subgroup $N$ of $G$. Suppose $\gamma \in N$. Then $\gamma=g a^{s} g^{-1}$ for some $g \in G$ and some $s$. Hence, $a^{s}=g^{-1} \gamma g \in N$ and from periodicity of $a$, it follows that also $a \in N$ as well as $a^{k} \in N$ for any $k$, because $p$ is prime. Then any element $h$ of $G$ also belongs to $N$ being of the form $h=\widetilde{h} a^{k}(\widetilde{h})^{-1}$, for some $k$, i.e., $N=G$. Thus, the group $G$ is simple. The discussion can be completed in the same way as in the case of Osin group. 
Example 6.3. In paper [23], Theorem III and its corollary, G. Higman, B. H. Neumann, and H. Neumann proved that any locally infinite countable group $G$ can be embedded into a countable group $G^{*}$ in which all elements except the unit element are conjugate to each other (see also [40]). The discussion above related Osin group remains valid for $G^{*}$ groups.

Let us remark that almost polycyclic group are residually finite (see e.g. [37, 5.4.17]) while the groups from these counterexamples are not residually finite, as it is clear by definition. That is why we would like to complete this section with the following question. Question. Suppose $G$ is a residually finite group and $\phi$ is its endomorphism with finite $R(\phi)$. Does $R(\phi)$ equal $S_{f}(\phi)$ ?

\section{Twisted CONJugacy SEPARATEness}

In fact, the notion of RP group is closely related with a generalization of the following well known notion.

Definition 7.1. A group $G$ is conjugacy separable if any pair $g, h$ of non-conjugate elements of $G$ are non-conjugate in some finite quotient of $G$.

It was proved that almost polycyclic groups are conjugacy separated $([34,15]$, see also [38, Ch. 4]). Also, residually finite recursively presented Burnside $p$-groups constructed by R. I. Grigorchuk [18] and by N. Gupta and S. Sidki [21] are shown to be conjugacy separable when $p$ is an odd prime in [44].

We can introduce the following notion, which coincides with the previous definition in the case $\phi=$ Id.

Definition 7.2. A group $G$ is $\phi$-conjugacy separable with respect to an automorphism $\phi: G \rightarrow G$ if any pair $g, h$ of non- $\phi$-conjugate elements of $G$ are non- $\bar{\phi}$-conjugate in some finite quotient of $G$ respecting $\phi$.

One gets immediately the following statement.

Theorem 7.3. Suppose, $R(\phi)<\infty$. Then $G$ is $\phi$-conjugacy separable if and only if $G$ is $\mathrm{RP}(\phi)$.

Proof. Indeed, let $F_{i j}: G \rightarrow K_{i j}$ distinguish $i$ th and $j$ th $\phi$-conjugacy classes, where $K_{i j}$ are finite groups, $i, j=1, \ldots, R(\phi)$. Let $F: G \rightarrow \oplus_{i, j} K_{i j}, F(g)=\sum_{i, j} F_{i j}(g)$, be the diagonal mapping and $K$ its image. Then $F: G \rightarrow K$ gives $\operatorname{RP}(\phi)$.

The opposite implication is evident.

Theorem 7.4. Let $F: \Gamma \rightarrow K$ be a morphism onto a finite group $K$ which separates two conjugacy classes of $\Gamma$ in $G \cdot t$. Then the restriction $F_{G}:=\left.F\right|_{G}: G \rightarrow \operatorname{Im}\left(\left.F\right|_{G}\right)$ separates the corresponding (by the bijection from Lemma 2.8) $\phi$-conjugacy classes in $G$.

Proof. First of all let us remark that $\operatorname{Ker}\left(F_{G}\right)$ is $\phi$-invariant. Indeed, suppose $F_{G}(g)=$ $F(g)=e$. Then

$$
F_{G}(\phi(g))=F(\phi(g))=F\left(t g t^{-1}\right)=F(t) F(t)^{-1}=e
$$

(the kernel of $F$ is a normal subgroup). 
Let $g t$ and $\tilde{g} t$ be some representatives of the mentioned conjugacy classes. Then

$$
\begin{gathered}
F\left(\left(h t^{n}\right) g t\left(h t^{n}\right)^{-1}\right) \neq F(\widetilde{g} t), \quad \forall h \in G, n \in \mathbb{Z}, \\
F\left(h t^{n} g t\right) \neq F\left(\widetilde{g} t h t^{n}\right), \quad \forall h \in G, n \in \mathbb{Z}, \\
F\left(h \phi^{n}(g) t^{n+1}\right) \neq F\left(\widetilde{g} \phi(h) t^{n+1}\right), \quad \forall h \in G, n \in \mathbb{Z}, \\
F\left(h \phi^{n}(g)\right) \neq F(\widetilde{g} \phi(h)), \quad \forall h \in G, n \in \mathbb{Z},
\end{gathered}
$$

in particular, $F\left(h g \phi\left(h^{-1}\right)\right) \neq F(\widetilde{g}) \forall h \in G$.

Theorem 7.5. Let some class of conjugacy separable groups be closed under taking semidirect products by $\mathbb{Z}$. Then this class consists of RP groups.

Proof. This follows immediately from Theorem 7.4 and Theorem 7.3.

As an application we obtain another proof of the main theorem for almost polycyclic groups.

Theorem 7.6. Any almost polycyclic group is an RP group.

Proof. The class of almost polycyclic groups is closed under taking semidirect products by $\mathbb{Z}$. Indeed, let $G$ be an almost polycyclic group. Then there exists a characteristic (polycyclic) subgroup $P$ of finite index in $G$. Hence, $P \rtimes \mathbb{Z}$ is a polycyclic normal group of $G \rtimes \mathbb{Z}$ of the same finite index.

Almost polycyclic groups are conjugacy separable $([34,15]$, see also $[38$, Ch. 4]). It remains to apply Theorem 7.5.

Remark 7.7. It is clear that this approach (i.e. the using of Theorem 7.5) can be extended to a number of situations (cf. [35]). The results of the present paper are generalized to the case of two endomorphisms and coincidences in [9]. The method of proof there is that of Sections $3-5$. The method of Section 7 does not work in this case.

In fact in [9] it is obtained in particular a generalization of the main result to the case of endomorphism. For the method of Sections $3-5$ the condition of finiteness of Reidemeister number is very important. On the other hand for the method of Section 7 the property of $\phi$ to be an automorphism is very important. Moreover, we can obtain the following final form of the twisted Burnside-Frobenius theorem for almost polycyclic and some other classes of groups.

Theorem 7.8 (Twisted Burnside-Frobenius theorem for $\phi$-conjugacy separable groups). Let $G$ be an almost polycyclic group (or, more generally, any group of a class under the hypothesis of Theorem 7.5, or even more generally, a $\phi$-conjugacy separable group). Then $R(\phi)=S_{f}(\phi)$ if one of these numbers is finite.

Proof. It remains to prove that $R(\phi)<\infty$ if $S_{f}(\phi)<\infty$. By the definition of a $\phi$ conjugacy separable group the Reidemeister classes of $\phi$ can be separated by maps to finite groups. Hence, taking representations of these finite groups and applying the twisted Burnside-Frobenius theorem to these groups we obtain that for any pair of Reidemeister classes there exists a function being a coefficient of a finite-dimensional unitary representation, which distinguish these classes. Hence, if $R(\phi)=\infty$, then there are infinitely many linear independent twisted invariant functions being coefficients of finite dimensional representations. But there are as many such functionals, as $S_{f}(\phi)$. 
Remark 7.9. As it is clear from the consideration in Section 6, the Osin group is not conjugacy separable, as well as its predecessors. In this relation it is natural to reformulate the question from in Section 6 in the following form.

Question. Suppose $G$ is a residually finite group and $\phi$ is its endomorphism with finite $R(\phi)$. Does this imply that $G$ is $\phi$-conjugacy separable?

Some other aspects of the twisted analog of Burnside-Frobenius theory are studied in [11].

\section{REFERENCES}

[1] J. Arthur and L. Clozel, Simple algebras, base change, and the advanced theory of the trace formula, Princeton University Press, Princeton, NJ, 1989. MR 90m:22041

[2] Charles W. Curtis and Irving Reiner, Representation theory of finite groups and associative algebras, Pure and Applied Mathematics, Vol. XI, Interscience Publishers, a division of John Wiley \& Sons, New York-London, 1962. MR MR0144979 (26 \#2519)

[3] J. Dixmier, $C^{*}$-algebras, North-Holland, Amsterdam, 1982.

[4] A. Fel'shtyn, Dynamical zeta functions, Nielsen theory and Reidemeister torsion, Mem. Amer. Math. Soc. 147 (2000), no. 699, xii+146. MR 2001a:37031

[5] A. Fel'shtyn and D. Gonçalves, Reidemeister numbers of Baumslag-Solitar groups, E-print arXiv:math.GR/0405590, 2004, (to appear in Algebra and Discrete Mathematics).

[6] A. Fel'shtyn and R. Hill, The Reidemeister zeta function with applications to Nielsen theory and a connection with Reidemeister torsion, K-Theory 8 (1994), no. 4, 367-393. MR 95h:57025

[7] _ Dynamical zeta functions, congruences in Nielsen theory and Reidemeister torsion, Nielsen theory and Reidemeister torsion (Warsaw, 1996), Polish Acad. Sci., Warsaw, 1999, pp. 77-116. MR 2001h:37047

[8] A. Fel'shtyn, F. Indukaev, and E. Troitsky, Twisted Burnside theorem for two-step torsion-free nilpotent groups, Preprint, Max-Planck-Institut für Mathematik, 2005.

[9] A. Fel'shtyn and E. Troitsky, Bitwisted Burnside-Frobenius theory, Preprint, 2005.

[10] _ - A twisted Burnside theorem for countable groups and Reidemeister numbers, Noncommutative Geometry and Number Theory (C. Consani and M. Marcolli, eds.), Vieweg, Braunschweig, 2006, pp. 141-154 (Preprint MPIM2004-65, math.RT/0606155).

[11] _ Twisted Frobenius theory, Preprint, 2006.

[12] A. Fel'shtyn, E. Troitsky, and A. Vershik, Twisted Burnside theorem for type $I_{1}$ groups: an example, Preprint 85, Max-Planck-Institut für Mathematik, 2004, (to appear in Math. Res. Lett., math.RT/0606161).

[13] A. L. Fel'shtyn, The Reidemeister number of any automorphism of a Gromov hyperbolic group is infinite, Zap. Nauchn. Sem. S.-Peterburg. Otdel. Mat. Inst. Steklov. (POMI) 279 (2001), no. 6 (Geom. i Topol.), 229-240, 250. MR 2002e:20081

[14] Alexander Fel'shtyn, Richard Hill, and Peter Wong, Reidemeister numbers of equivariant maps, Topology Appl. 67 (1995), no. 2, 119-131. MR MR1362078 (96j:58139)

[15] E. Formanek, Conjugacy separability in polycyclic groups, J. Algebra 42 (1976), 1-10.

[16] D. Gonçalves and P. Wong, Twisted conjugacy classes in exponential growth groups, Bull. London Math. Soc. 35 (2003), no. 2, 261-268. MR 2003j:20054

[17] Daciberg L. Gonçalves, The coincidence Reidemeister classes of maps on nilmanifolds, Topol. Methods Nonlinear Anal. 12 (1998), no. 2, 375-386. MR MR1701269 (2000d:55004)

[18] R.I. Grigorchuk, On Burnside's problem on periodic groups, Funct. Anal. Appl. 14 (1980), 41-43.

[19] Mikhael Gromov, Groups of polynomial growth and expanding maps, Inst. Hautes Études Sci. Publ. Math. (1981), no. 53, 53-73. MR MR623534 (83b:53041)

[20] A. Grothendieck, Formules de Nielsen-Wecken et de Lefschetz en géométrie algébrique, Séminaire de Géométrie Algébrique du Bois-Marie 1965-66. SGA 5, Lecture Notes in Math., vol. 569, SpringerVerlag, Berlin, 1977, pp. 407-441.

[21] N. Gupta and S. Sidki, On the Burnside problem for periodic groups, Math. Z. 182 (1983), 385-388. 
[22] Ph. Hall, A characteristic property of soluble groups, J. London Math. Soc. 12 (1937), 198-200.

[23] Graham Higman, B. H. Neumann, and Hanna Neumann, Embedding theorems for groups, J. London Math. Soc. 24 (1949), 247-254. MR MR0032641 (11,322d)

[24] B. Jiang, Lectures on Nielsen fixed point theory, Contemp. Math., vol. 14, Amer. Math. Soc., Providence, RI, 1983.

[25] Bo ju Jiang, A characterization of fixed point classes, Fixed point theory and its applications (Berkeley, CA, 1986), Contemporary Math., no. 72, Amer. Math. Soc., 1988, pp. 157-160.

[26] A. A. Kirillov, Elements of the theory of representations, Springer-Verlag, Berlin Heidelberg New York, 1976.

[27] A. G. Kurosh, The theory of groups, Translated from the Russian and edited by K. A. Hirsch. 2nd English ed. 2 volumes, Chelsea Publishing Co., New York, 1960. MR MR0109842 (22 \#727)

[28] G. Levitt, On the automorphism group of generalised Baumslag-Solitar groups, E-print arxiv:math.GR/0511083, 2005.

[29] G. Levitt and M. Lustig, Most automorphisms of a hyperbolic group have very simple dynamics., Ann. Scient. Éc. Norm. Sup. 33 (2000), 507-517.

[30] A. I. Mal'cev, On the faithful representations of infinite groups by matrices, Mat. Sb. (NS) 8(50) (1940), 405-422, (in Russian. English translation: Amer. Math. Soc. Transl. (2), 45 (1965), 1-18).

[31] B. H. Neumann, Groups with finite classes of conjugate elements, Proc. London Math. Soc. (3) 1 (1951), 178-187. MR MR0043779 (13,316c)

[32] A. Yu. Ol'shanskiǔ, Geometry of defining relations in groups, Mathematics and its Applications (Soviet Series), vol. 70, Kluwer Academic Publishers Group, Dordrecht, 1991, Translated from the 1989 Russian original by Yu. A. Bakhturin. MR MR1191619 (93g:20071)

[33] D. Osin, Small cancellations over relatively hyperbolic groups and embedding theorems, Arxiv e-print math.GR/0411039, 2004.

[34] V. N. Remeslennikov, Conjugacy in polycyclic groups. (Russian), Algebra i Logika 8 (1969), 712-725.

[35] L. Ribes, D. Segal, and P. A. Zalesskii, Conjugacy separability and free products of groups with cyclic amalgamation, J. London Math. Soc. (2) 57 (1998), no. 3, 609-628.

[36] Derek J. S. Robinson, Finiteness conditions and generalized soluble groups. Part 1, Springer-Verlag, New York, 1972, Ergebnisse der Mathematik und ihrer Grenzgebiete, Band 62. MR MR0332989 (48 \#11314)

[37] _ . A course in the theory of groups, second ed., Graduate Texts in Mathematics, vol. 80, Springer-Verlag, New York, 1996. MR MR1357169 (96f:20001)

[38] Daniel Segal, Polycyclic groups, Cambridge Tracts in Mathematics, no. 82, Cambridge University Press, Cambridge, 1983.

[39] Jean-Pierre Serre, Linear representations of finite groups, Springer-Verlag, New York, 1977, Translated from the second French edition by Leonard L. Scott, Graduate Texts in Mathematics, Vol. 42. MR MR0450380 (56 \#8675)

[40] _ Trees, Springer Monographs in Mathematics, Springer-Verlag, Berlin, 2003, Translated from the French original by John Stillwell, Corrected 2nd printing of the 1980 English translation. MR MR1954121 (2003m:20032)

[41] Salahoddin Shokranian, The Selberg-Arthur trace formula, Lecture Notes in Mathematics, vol. 1503, Springer-Verlag, Berlin, 1992, Based on lectures by James Arthur. MR MR1176101 (93j:11029)

[42] Jennifer Taback and Peter Wong, Twisted conjugacy and quasi-isometry invariance for generalized solvable Baumslag-Solitar groups, E-print arxiv:math.GR/0601271, 2006.

[43] E. Troitsky, Noncommutative Riesz theorem and weak Burnside type theorem on twisted conjugacy, Funct. Anal. Pril. 40 (2006), no. 2, 44-54, In Russian, English translation: Funct. Anal. Appl. 40 (2006), No. 2, 117-125 (Preprint 86 (2004), Max-Planck-Institut für Mathematik, math.OA/0606191).

[44] J. S. Wilson and P. A. Zalesskii, Conjugacy separability of certain torsion groups, Arch. Math. (Basel) 68 (1997), no. 6, 441-449. 
Instytut Matematyki, Uniwersytet Szczecinski, Ul. Wielkopolska 15, 70-451 Szczecin, Poland and Department of Mathematics, Boise State University, 1910 University Drive, BoISE, IDAHO, 83725-155, USA

E-mail address: felshtyn@diamond.boisestate.edu, felshtyn@mpim-bonn.mpg.de

Dept. of Mech. and Math., Moscow State University, 119992 GSP-2 Moscow, Russia

E-mail address: troitsky@mech.math.msu.su

URL: http://mech.math.msu.su/ ${ }^{\sim}$ troitsky 